MIFP-05/02

UUITP-16/02

hep-th/0211113

\title{
Superspace Formulation of 4D Higher Spin Gauge Theory
}

\author{
J. Engquist ${ }^{1}$, E. Sezgin ${ }^{2}$ and P. Sundell ${ }^{3}$ \\ 1,3 Department of Theoretical Physics, Uppsala University, \\ Box 803, 75108 Uppsala, Sweden \\ ${ }^{2}$ George P. and Cynthia W. Mitchell Institute for Fundamental Physics, \\ Texas A EM University, College Station, TX 77843-4242, USA
}

\begin{abstract}
Interacting $\mathrm{AdS}_{4}$ higher spin gauge theories with $\mathcal{N} \geq 1$ supersymmetry so far have been formulated as constrained systems of differential forms living in a twistor extension of $4 \mathrm{D}$ spacetime. Here we formulate the minimal $\mathcal{N}=1$ theory in superspace, leaving the internal twistor space intact. Remarkably, the superspace constraints have the same form as those defining the theory in ordinary spacetime. This construction generalizes straightforwardly to higher spin gauge theories $\mathcal{N} \geq 2$ supersymmetry.
\end{abstract}




\section{INTRODUCTION}

The $\mathcal{N}=8$ massless higher spin (HS) gauge theory in $D=4$ [1] is expected to arise as a consistent truncation of $\mathrm{M}$ theory on $A d S_{4} \times S^{7}$ in an unbroken phase describing the theory at high energies $[2,3]$. Various $\mathcal{N}=1,2,4$ HS gauge theories can arise in less symmetric compactifications of M theory [4]. In this paper we shall only discuss massless HS gauge theories theories, though in general massive fields are needed for embedding in $\mathrm{M}$ theory. From the $3 \mathrm{~d}$ holographic point of view, the unbroken phase of $\mathrm{M}$ theory on $A d S_{4} \times S^{7}$ has been conjectured to correspond to an $S U(N)$ invariant free singleton theory [2,3], and the massless sector to an $O\left(N^{2}-1\right)$ vector model [5]. Similar ideas in the context of the Type IIB theory on $A d S_{5} \times S^{5}$ have been discussed in $[6,7,8,9,3]$.

Interacting $\mathrm{AdS}_{4} \mathrm{HS}$ gauge theories with $\mathcal{N} \geq 1$ supersymmetry so far have been formulated as constrained systems of differential 0 -forms and 1-forms living in the product of $4 \mathrm{D}$ spacetime with an internal twistor space [1]. In this formulation the local HS symmetries are realized as internal gauge symmetries, and spacetime diffeomorphisms are incorporated into the gauge group as local field dependent translations. By expanding in curvatures [10] it is possible to obtain the non-linear field equations in a manifestly reparametrization invariant form [11]. For a better understanding of the geometrical structures underlying HS gauge theories it would be desirable to extend the diffeomorphism symmetry as to include additional generators of the HS symmetry algebra. A natural first step in this direction is to reformulate the HS gauge theories in superspace with manifest superdiffeomorphism invariance. The superspace formulation of free $4 \mathrm{D}$ conformal HS gauge theories with $\mathcal{N} \geq 1$ supersymmetry has been given in [8]. The superspace formulation of the linearized $\mathrm{AdS}_{4}$ HS gauge theory based on a particular $\mathcal{N}=2$ algebra has been given in [12].

In this paper we extend the integrable systems describing interacting $\mathrm{AdS}_{4} \mathrm{HS}$ gauge theories, by replacing the $4 \mathrm{D}$ spacetime by a $D=(4 \mid 4 \mathcal{N})$ superspace with $4 \mathcal{N}$ anticommuting $\theta$-coordinates. This introduces extra spinorial directions in the 1-forms as well as $\theta$-dependence in all component fields. On the other hand, there are also new constraints coming from projections of the differential form constraints in the new spinorial directions. As a result, each supermultiplet in the spectrum is described by a single constrained superfield, and we arrive at a superspace description of $\mathrm{AdS}_{4} \mathrm{HS}$ gauge theory which is equivalent to the formulation in ordinary spacetime. This equivalence exploits a basic property of integrable systems which ensures that adding extra coordinates does not affect the basic dynamics described by the system, as we shall discuss at the end of Section 2.1.

The structure of the paper is as follows: In Section 2, we formulate the minimal $h s(1 \mid 4)$ theory in the product of $\mathcal{N}=1, D=4$ superspace with the internal twistor 
space. Furthermore, we eliminate the twistor variable using a curvature expansion. In Section 3, we obtain the superspace constraints on the physical superfields, and in particular show that they yield the correct spectrum of the theory. In Section 4 , we summarize our results, and comment on how to extend the formalism to $\mathcal{N} \geq 2$ theories in $D=4$ and the $\mathcal{N}=4$ HS gauge theory in $D=5$.

\section{The $h s(1 \mid 4)$ Gauge Theory in Superspace}

In this section we first introduce the basic properties of the minimal $\mathcal{N}=1, D=$ 4 HS gauge theory, namely the underlying HS symmetry algebra $h s(1 \mid 4)$ and its massless spectrum [13, 4]. Next we give the constraints which describe the full equations of motion in superspace, and give a formal argument for their equivalence to the formulation in ordinary spacetime. We then generalize the covariant curvature expansion of the constraints $[10,11]$ to superspace.

The formulation of higher spin dynamics in ordinary 4D spacetime follows from constraints on certain one-form and zero-form master fields which live in the product of spacetime with an internal twistor space [1] (our starting point is summarized in [4]). A key property of these constraints is that all curvature components with at least one spacetime direction are set equal to zero. Hence the form of the constraints do not depend on the details of the spacetime manifold. In fact, this implies that the constraints equivalently can be formulated on extended spacetime manifolds with extra coordinates, such as superspace.

\subsection{The Closed Form of the Constraints}

The minimal $\mathcal{N}=1, D=4 \mathrm{HS}$ theory is based on the HS algebra $h s(1 \mid 4)$ whose maximal finite-dimensional subalgebra is $O S p(1 \mid 4)$. The spectrum of massless physical fields of the $h s(1 \mid 4)$ gauge theory is given by the symmetric product of two $O S p(1 \mid 4)$ singletons, which is given in Table 1. The spectrum is an UIR of $h s(1 \mid 4)$ and decomposes into a tower of $O S p(1 \mid 4)$ multiplets labelled by a level index

$$
(\ell, j)=\left\{\begin{array}{ll}
\ell=0,1,2, \ldots & \text { for } j=0 \\
\ell=-1,0,1, \ldots & \text { for } j=1 / 2
\end{array},\right.
$$

with maximal spin

$$
s_{\max }=2 \ell+2+j
$$


In particular, the spectrum contains a scalar multiplet at level $(-1,1 / 2)$ and a supergravity multiplet at level $(0,0)$.

To obtain an $\mathcal{N}=1$ superspace formulation of the $h s(1 \mid 4)$ theory we start from the formulation in ordinary $4 \mathrm{D}$ spacetime $[1,4]$ and make everywhere the replacement

$$
x^{m} \rightarrow X^{M}=\left(x^{m}, \theta^{\mu}, \bar{\theta}^{\dot{\mu}}\right), \quad m=0, \ldots, 3, \quad \mu, \dot{\mu}=1,2 .
$$

The resulting theory is described in terms of a master one-form

$$
\widehat{A}=d X^{M} \widehat{A}_{M}(X, Z ; Y, \xi, \eta)+d Z^{\underline{\alpha}} \widehat{A}_{\underline{\alpha}}(X, Z ; Y, \xi, \eta)
$$

and master zero-form

$$
\widehat{\Phi}=\widehat{\Phi}(X, Z ; Y, \xi, \eta)
$$

where $Z$ stands for $Z^{\underline{\alpha}}=\left(z^{\alpha}, \bar{z}^{\dot{\alpha}}\right)$ and $Y$ for $Y^{\underline{\alpha}}=\left(y^{\alpha}, \bar{y}^{\dot{\alpha}}\right)$, which are bosonic oscillators that generate the following associative algebra of Weyl-ordered functions:

$$
\begin{aligned}
\widehat{f}(Z ; Y) \star \widehat{g}(Z ; Y) & =\widehat{f}(Z ; Y) \exp \left(i \overleftarrow{\partial}^{(+) \alpha} \vec{\partial}_{\alpha}^{(-)}+i \overleftarrow{\partial}^{(-) \dot{\alpha}} \vec{\partial}_{\dot{\alpha}}^{(+)}\right) \widehat{g}(Z ; Y) \\
\partial_{\alpha}^{( \pm)} & =\partial / \partial z^{\alpha} \pm \partial / \partial y^{\alpha}
\end{aligned}
$$

The quantities $\xi$ and $\eta$ are fermionic oscillators obeying

$$
\xi \star \xi=1, \quad \xi \star \eta=\xi \eta=-\eta \xi=\eta \star \xi . \quad \eta \star \eta=1 .
$$

The master fields have $(Z ; Y, \xi, \eta)$ expansions obeying the following conditions

$$
\begin{array}{llll}
\tau(\widehat{A})=-\widehat{A}, & \widehat{A}^{\dagger}=-\widehat{A}, & \epsilon(\widehat{A})=0, \\
\tau(\widehat{\Phi})=\bar{\pi}(\widehat{\Phi}), & \widehat{\Phi}^{\dagger}=\pi(\widehat{\Phi}) \star \Gamma, & \epsilon(\widehat{\Phi})=0,
\end{array}
$$

where $\Gamma=i \xi \eta, \epsilon$ denotes the Grassmann parity, and $\tau, \pi$ and $\bar{\pi}$ are maps that commute with the exterior derivative and act on functions of $X$ and the oscillators as follows 


$$
\begin{aligned}
\tau(\widehat{f}(X, z, \bar{z} ; y, \bar{y}, \xi, \eta)) & \equiv \widehat{f}(X,-i z,-i \bar{z} ; i y, i \bar{y}, i \xi,-i \eta) \\
\pi(\widehat{f}(X, z, \bar{z} ; y, \bar{y}, \xi, \eta)) & \equiv \widehat{f}(X,-z, \bar{z} ;-y, \bar{y}, \xi, \eta) \\
\bar{\pi}(\widehat{f}(X, z, \bar{z} ; y, \bar{y}, \xi, \eta)) & \equiv \widehat{f}(X, z,-\bar{z} ; y,-\bar{y}, \xi, \eta)
\end{aligned}
$$

The $h s(1 \mid 4)$-valued connection and its quasi-adjoint representation are obtained by setting $Z=0$ in (2.8) and (2.9), respectively.

We propose that the full $\mathcal{N}=1$ superspace formulation of the $h s(1 \mid 4)$ theory is given by the following curvature constraints:

$$
\begin{aligned}
\widehat{D} \widehat{\Phi} & =0 \\
\widehat{F} & =\frac{i}{4} d z^{\alpha} \wedge d z_{\alpha} \mathcal{V}(\widehat{\Phi} \star \kappa \Gamma)+\frac{i}{4} d \bar{z}^{\dot{\alpha}} \wedge d \bar{z}_{\dot{\alpha}} \overline{\mathcal{V}}\left(\widehat{\Phi} \star \kappa^{\dagger}\right)
\end{aligned}
$$

where

$$
\begin{aligned}
\widehat{D} \widehat{\Phi} & \equiv d \widehat{\Phi}+\widehat{\Phi} \star \bar{\pi}(\widehat{A})-\widehat{A} \star \widehat{\Phi} \\
\widehat{F} & \equiv d \widehat{A}+\widehat{A} \star \widehat{A}
\end{aligned}
$$

and

$$
d=d X^{M} \partial_{M}+d Z^{\underline{\alpha}} \partial_{\underline{\alpha}}
$$

We stress that the pull-back of the constraints (2.13) and (2.14) from $(X, Z)$-space to $(x, Z)$-space yields back the original formulation of the $h s(1 \mid 4)$ theory $[1,4]$ (there is a change of sign in $\mathcal{V}(\widehat{\Phi} \star \kappa)$, and hence in $\widehat{\Phi}$, due the change between left and right acting exterior derivatives).

The basic properties of (2.13) and (2.14) are that they are integrable and consistent with the $\tau$ and reality conditions on the master fields given in (2.8) and (2.9). The integrability of the constraints implies their invariance under the following gauge transformations:

$$
\delta \widehat{A}=d \widehat{\epsilon}+\widehat{\epsilon} \star \widehat{A}-\widehat{A} \star \widehat{\epsilon}, \quad \delta \widehat{\Phi}=\widehat{\epsilon} \star \widehat{\Phi}-\widehat{\Phi} \star \bar{\pi}(\widehat{\epsilon})
$$


where the gauge parameter $\widehat{\epsilon}(X, Z ; Y, \xi, \eta)$ obeys (2.8). A superdiffeomorphism with parameter $\xi=\xi^{M}(X) \partial_{M}$ is equivalent to a field dependent gauge transformations with parameter $\widehat{\epsilon}=i_{\xi} \widehat{A}$.

By fixing a gauge and making use of a subset of the constraints one can eliminate the $Z$-dependence and thus reduce the constraints to an equivalent but smaller set of constraints in superspace. This yields ordinary superspace constraints, from which one can obtain the $\theta$-expansions of the master fields, as we shall show in Section 3. It is also possible to eliminate the $X$ dependence, and thus obtain an equivalent formulation entirely in $Z$-space [14]. This yields a non-standard form of the dynamical field equations, that could be a convenient framework for obtaining exact solutions.

The equivalences between the formulations in superspace, ordinary spacetime and the $Z$-space, follow from the integrability which implies that given $\widehat{\Phi}(0,0 ; Y, \xi, \eta)$ it is possible to determine $\widehat{\Phi}$ and $\widehat{A}$ for finite $X$ and $Z$ by making use of the constraints and by fixing a gauge. Hence, the formulations in superspace, ordinary spacetime as well as in $Z$-space are formulations on different slices of the full $(X, Z)$-space obtained by various (partial) gauge fixings.

From the ordinary spacetime point of view, the quantity $\widehat{\Phi}(0,0 ; Y, \xi, \eta)$ describes all the dynamical fields as well as all of their spacetime derivatives that are nonvanishing on-shell [14]. In going to the superspace formulation, the same quantity describes all the dynamical superfields as well as all of their superderivatives that are non-vanishing on-shell, implying that the two formulations are equivalent. As we shall show, there will be one independent superfield for each multiplet in the spectrum listed in Table 1.

\subsection{Curvature Expansion of the Constraints}

The $Z$-dependence of the master fields can be determined in terms of

$$
\left.\Phi \equiv \widehat{\Phi}\right|_{Z=0},\left.\quad A_{M} \equiv \widehat{A}_{M}\right|_{Z=0}
$$

by integrating the components of (2.13) and (2.14) that have at least one $d z^{\alpha}$ or $d \bar{z}^{\dot{\alpha}}$ component. The solution can be given as a curvature expansion in powers of $\Phi$ :

$$
\widehat{\Phi}=\sum_{i=1}^{\infty} \widehat{\Phi}^{(i)},\left.\quad \widehat{\Phi}^{(i)}\right|_{Z=0}= \begin{cases}\Phi, & i=1 \\ 0, & i=2,3, \ldots\end{cases}
$$




$$
\begin{aligned}
& \widehat{A}_{M}=\sum_{i=0}^{\infty} \widehat{A}_{M}^{(i)},\left.\quad \widehat{A}_{M}^{(i)}\right|_{Z=0}=\left\{\begin{array}{ll}
A_{M}, & i=0 \\
0, & i=1,2,3, \ldots
\end{array},\right. \\
& \widehat{A}_{\alpha}=\sum_{i=0}^{\infty} \widehat{A}_{\alpha}^{(i)},
\end{aligned}
$$

where $\widehat{\Phi}^{(i)}, \widehat{A}_{\alpha}^{(i)}$ and $\widehat{A}_{M}^{(i)}$ are $i$ th order in $\Phi$. The iterative formulae for $\widehat{\Phi}^{(i)}, \widehat{A}_{\alpha}^{(i)}$ and $\widehat{A}_{M}^{(i)}$ are direct generalizations of those given in [11] in the case of ordinary spacetime. In particular, the superspace one-form is given by

$$
\widehat{A}_{M}=\frac{1}{1+\widehat{L}^{(1)}+\widehat{L}^{(2)}+\widehat{L}^{(3)}+\cdots} A_{M}
$$

where the linear operators $\widehat{L}^{(i)}$ are given in [11]. The operator $\widehat{L}^{(1)}$, which will be needed below, takes the form

$$
\begin{aligned}
\widehat{L}^{(1)}(\widehat{f})=\frac{1}{2} \int_{0}^{1} \frac{t^{\prime} d t^{\prime} d t}{t}( & {\left[\left(\Phi\left(-t^{\prime} z, \bar{y}, \xi, \eta\right) \kappa\left(t^{\prime} z, y\right) z^{\alpha}\right) \star \Gamma, \frac{\partial \widehat{f}}{\partial y^{\alpha}}\right]_{\star} } \\
& \left.-\left[\Phi\left(y, t^{\prime} \bar{z}, \xi, \eta\right) \bar{\kappa}\left(t^{\prime} \bar{z}, \bar{y}\right) \bar{z}^{\dot{\alpha}}, \frac{\partial \widehat{f}}{\partial \bar{y}^{\dot{\alpha}}}\right]_{\star}\right)_{Z \rightarrow t Z}
\end{aligned}
$$

Upon inserting the expressions for $\widehat{\Phi}$ and $\widehat{A}_{M}$ in terms of $\Phi$ and $A_{M}$ into the remaining components of (2.13) and (2.14), i.e. $\widehat{D}_{M} \widehat{\Phi}=0$ and $\widehat{F}_{M N}=0$, we obtain curvature constraints in $\mathcal{N}=1$ superspace. To analyze these constraints we need to convert the curved index $M$ into the flat Lorentz index $A$, which can be decomposed covariantly into Lorentz vector and spinor indices. In order to do this, one first identifies the exact form of the local Lorentz structure group [15]. Following [11], we decompose $A_{M}$ as follows:

$$
A_{M}=E_{M}+\Omega_{M}+W_{M}+K_{M}
$$

where $W_{M}$ contains the higher spin gauge fields (see (3.3) below), and ${ }^{1}$

\footnotetext{
${ }^{1}$ The notation here differs from that in [11] where $E$ denotes the $S O(3,2)$ valued gauge field, $E=e+\omega$ where $e$ is the vierbein and $\omega$ the Lorentz connection, and $\Omega$ denotes the rigid $S O(3,2)$ gauge field in the $\mathrm{AdS}_{4}$ background.
} 


$$
\begin{aligned}
& E_{M}=i\left(E_{M}^{\alpha} Q_{\alpha}+E_{M}^{\dot{\alpha}} Q_{\dot{\alpha}}+E_{M}^{\alpha \dot{\alpha}} P_{\alpha \dot{\alpha}}\right) \\
& \Omega_{M}=\frac{i}{4}\left(\Omega_{M}^{\alpha \beta} M_{\alpha \beta}+\Omega_{M}^{\dot{\alpha} \dot{\beta}} M_{\dot{\alpha} \dot{\beta}}\right)
\end{aligned}
$$

Here $\Omega_{M}$ is the spin connection and $E_{M}$ define the supervielbein

$$
E_{M}^{A}=\left(E_{M}^{a}, E_{M}^{\alpha}, E_{M}^{\dot{\alpha}}\right)
$$

In (2.25) we have also separated out

$$
K_{M}=-\left.i \Omega_{M}^{\alpha \beta}\left(\widehat{A}_{\alpha} \star \widehat{A}_{\beta}\right)\right|_{Z=0}-\text { h.c. }
$$

which has the effect of making the constraints manifestly Lorentz covariant [15] (see also [11]). As a result the dependence of the constraints on the spin connection is through either the covariant derivative

$$
\nabla=d X^{M}\left(\partial_{M}+\Omega_{M}\right)
$$

or the Riemann curvature two-form $R=d \Omega+\Omega \star \Omega$. We further define the inverse supervielbein $E_{A}^{M}$ by $E_{A}^{M} E_{M}^{B}=\delta_{A}^{B}$ and use the notation

$$
E=i d X^{M} E_{M}^{A} Q_{A}=i E^{A} Q_{A}, \quad Q_{A}=\left(P_{a}, Q_{\alpha}, Q_{\dot{\alpha}}\right)
$$

The desired manifestly Lorentz invariant form of the superspace constraints read:

$$
\begin{aligned}
\mathcal{R}_{A B}+\mathcal{F}_{A B}= & -2 W_{[A} \star W_{B]}+i \sum_{\substack{i+j=2 \\
i, j \geq 1}}^{\infty}\left(\left.R_{A B}^{\alpha \beta}\left(\widehat{A}_{\alpha}^{(i)} \star \widehat{A}_{\beta}^{(j)}\right)\right|_{Z=0}+\text { h.c. }\right) \\
& -\left.2 \sum_{i+j=1}^{\infty}\left((i \widehat{Q}+\widehat{W})_{[A}^{(i)} \star(i \widehat{Q}+\widehat{W})_{B]}^{(j)}\right)\right|_{Z=0}
\end{aligned}
$$




$$
\nabla_{A} \Phi+i \Phi \star \bar{\pi}\left(Q_{A}\right)-i Q_{A} \star \Phi=-\left.\sum_{\substack{i+j=2 \\ i \geq 1}}^{\infty}\left(\widehat{\Phi}^{(i)} \star \bar{\pi}\left((i \widehat{Q}+\widehat{W})_{A}^{(j)}\right)-(i \widehat{Q}+\widehat{W})_{A}^{(j)} \star \widehat{\Phi}^{(i)}\right)\right|_{Z=0}
$$

where $[A B]$ denotes graded symmetrization and we have made the following definitions: the $O S p(1 \mid 4)$ covariant gravitational curvature $\mathcal{R}$ is defined by

$$
\begin{aligned}
\mathcal{R} & \equiv d(E+\Omega)+(E+\Omega) \star(E+\Omega) \\
& =\nabla E+d \Omega+\Omega \star \Omega+E \star E \\
& =i\left(\mathcal{R}^{\alpha} Q_{\alpha}+\mathcal{R}^{\dot{\alpha}} Q_{\dot{\alpha}}+\mathcal{R}^{\alpha \dot{\alpha}} P_{\alpha \dot{\alpha}}+\frac{1}{4} \mathcal{R}^{\alpha \beta} M_{\alpha \beta}+\frac{1}{4} \mathcal{R}^{\dot{\alpha} \dot{\beta}} M_{\dot{\alpha} \dot{\beta}}\right),
\end{aligned}
$$

with components

$$
\begin{aligned}
\mathcal{R}^{\alpha} & =T^{\alpha}+2 E^{\dot{\alpha}} \wedge E_{\dot{\alpha}}{ }^{\alpha}, \\
\mathcal{R}^{\alpha \dot{\alpha}} & =T^{\alpha \dot{\alpha}}-\frac{i}{2} E^{\alpha} \wedge E^{\dot{\alpha}}, \\
\mathcal{R}^{\alpha \beta} & =R^{\alpha \beta}-i E^{\alpha} \wedge E^{\beta}+4 E^{\alpha \dot{\alpha}} \wedge E_{\dot{\alpha}}{ }^{\beta}
\end{aligned}
$$

where the superspace torsion $T^{A}$ and Riemann curvature $R^{\alpha \beta}$ are defined by

$$
\begin{aligned}
T^{A} & =\nabla E^{A}, \\
R^{\alpha \beta} & =d \Omega^{\alpha \beta}+\Omega^{\alpha \gamma} \wedge \Omega_{\gamma}{ }^{\beta} .
\end{aligned}
$$

Rigid $\mathrm{AdS}_{4}$ superspace is obtained by setting $\mathcal{R}=0$. The $O S p(1 \mid 4)$ covariant higher spin curvature $\mathcal{F}$ is defined by

$$
\begin{aligned}
\mathcal{F} & =d W+(E+\Omega) \star W+W \star(E+\Omega) \\
& =\nabla W+E \star W+W \star E,
\end{aligned}
$$

and has the following components

$$
\mathcal{F}_{A B}=2 \nabla_{[A} W_{B]}+T_{A B}^{C} W_{C}-2 i Q_{[A} \star W_{B]}-2 i W_{[A} \star Q_{B]},
$$




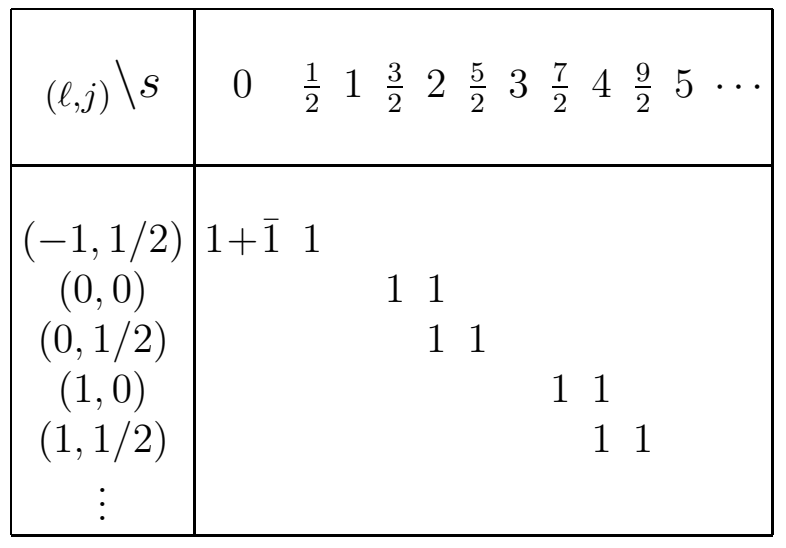

Table 1: The spectrum of massless physical fields of the minimal $\mathcal{N}=1, D=4$ higher spin gauge theory arranged into levels of $\mathcal{N}=1$ supermultiplets labelled by $(\ell, j)$ and with $s_{\max }=2 \ell+2+j$.

where $[A B]$ denotes graded symmetrization. Finally, the quantity $\widehat{A}_{\alpha}$ in $(2.32)$ is defined in (2.4).

The constraints (2.32) and (2.33) are invariant under gauge transformations with $h s(1 \mid 4)$-valued superfield parameters and under superspace diffeomorphisms. The analysis of the symmetries is analogous to the case of ordinary spacetime; see, for example, [11] for a discussion and the explicit form of the gauge transformations.

The constraints (2.32) and (2.33) define the full $h s(1 \mid 4)$ gauge theory in superspace. As explained in Section 2.1, this superspace formulation is equivalent to the formulation in ordinary spacetime. To verify this directly, one begins with the restriction of (2.32) and (2.33) to the bosonic submanifold, which, by construction, yields the curvature constraints in ordinary spacetime described in [4]. Thus, in order to verify the equivalence between the two formulations it suffices to show that the superspace formulation does not contain any additional propagating degrees of freedom nor any new constraints. This can be analyzed in a manifestly superspace covariant expansion in which both $\Phi$ and the higher spin gauge fields $W_{M}$ are weak fields, while the gauge fields of the supergravity multiplet are treated exactly [11]. If the leading order does not yield any new degrees of freedom or constraints, then this must hold to all orders in the weak field expansion. Indeed this will be shown to be the case in the next section. 


\section{Analysis of Superspace Constraints}

In this section we analyze the superspace constraints (2.32) and (2.33) in the first order in the weak field expansion, in which we treat $\Phi$ and $W_{M}$ as weak fields while the supervielbein $E_{M}$ contains information of the full ordinary $\mathcal{N}=1$ supergravity theory (without higher derivative corrections). Indeed, we will find the superspace constraints that describe the ordinary on-shell $\mathcal{N}=1, D=4$ supergravity with cosmological constant. Moreover, we will find the constraints on the superfields describing the scalar and the higher spin multiplets in the first order in the weak fields.

\subsection{WEAK FIELD EXPANSION}

Assuming that both $\Phi$ and the higher spin gauge fields $W_{M}$ are weak fields, the leading contributions to the superspace constraints (2.32) and (2.33) are

$$
\begin{aligned}
\mathcal{R}+\mathcal{F}^{(1)}= & -\left.\left\{E, \widehat{E}^{(1)}\right\}_{\star}\right|_{Z=0}=\left.\left\{E, \widehat{L}^{(1)}(E)\right\}_{\star}\right|_{Z=0} \\
= & -\left(E^{\dot{\alpha}} \wedge E_{\dot{\alpha}}{ }^{\alpha} \frac{\partial}{\partial y^{\alpha}} \frac{\partial}{\partial \xi}+i E^{\alpha \dot{\alpha}} \wedge E_{\dot{\alpha}}{ }^{\beta} \frac{\partial}{\partial y^{\alpha}} \frac{\partial}{\partial y^{\beta}}\right) \Phi(y, 0, \xi, \eta) \\
& -\left(E^{\alpha} \wedge E_{\alpha}{ }^{\dot{\alpha}} \frac{\partial}{\partial \bar{y}^{\dot{\alpha}}} \frac{\partial}{\partial \xi}+i E^{\dot{\alpha} \alpha} \wedge E_{\alpha} \dot{\beta} \frac{\partial}{\partial \bar{y}^{\dot{\alpha}}} \frac{\partial}{\partial \bar{y}^{\dot{\beta}}}\right)(\Phi(0, \bar{y}, \xi, \eta) \star \Gamma),
\end{aligned}
$$

and

$$
\nabla_{A} \Phi+i \Phi \star \bar{\pi}\left(Q_{A}\right)-i Q_{A} \star \Phi=0
$$

where the higher spin field strength $\mathcal{F}^{(1)}$ is given by (2.41) with $T_{A B}{ }^{C}$ set equal to the rigid $\mathrm{AdS}_{4}$ superspace torsion and $\widehat{L}^{(1)}$ is defined in (2.24). The supervielbein and the spin connection describe a curved superspace, which as we shall see is pure supergravity in the leading order. The integrability of (3.1) and (3.2) holds modulo

terms which involve the supergravity field strengths, which are given in (3.16) and (3.17) below, times other weak fields.

We proceed by decomposing $W_{M}$ and $\Phi$ into levels as follows [4]

$$
W_{M}=W_{M}^{(0,1 / 2)}+\sum_{\ell \geq 1}\left(W_{M}^{(\ell, 0)}+W_{M}^{(\ell, 1 / 2)}\right)
$$




$$
\Phi=\Phi^{(-1,1 / 2)}+\sum_{\ell \geq 0}\left(\Phi^{(\ell, 0)}+\Phi^{(\ell, 1 / 2)}\right)
$$

where

$$
\begin{aligned}
W_{M}^{(\ell, j)} & =\sum_{p+q+r=4 \ell+2+2 j} W_{M, r}^{(\ell, j)}(p, q) \xi^{r} \eta^{2 j} \\
\Phi^{(\ell, j)} & =C^{(\ell, j)}+\pi\left(\left(C^{(\ell, j)}\right)^{\dagger}\right) \star \Gamma \\
C^{(\ell, j)} & =\sum_{\substack{q-p-r \\
=4 \ell+3+2 j}} \Phi_{r}^{(\ell, j)}(p, q) \xi^{r} \eta^{1-2 j} .
\end{aligned}
$$

and $(p, q)$ refers to the $y$ and $\bar{y}$ expansion as defined in (A.11). Since the adjoint and quasi-adjoint actions of $Q_{A}$ on $W^{(\ell, j)}$ and $\Phi^{(\ell, j)}$ preserve the level index, it follows that the constraints (3.1) and (3.2) split into a separate set of constraints for each level:

$$
\begin{gathered}
\mathcal{R}=-\left(E^{\dot{\alpha}} \wedge E_{\dot{\alpha}}{ }^{\alpha} \frac{\partial}{\partial y^{\alpha}} \frac{\partial}{\partial \xi}+i E^{\alpha \dot{\alpha}} \wedge E_{\dot{\alpha}}{ }^{\beta} \frac{\partial}{\partial y^{\alpha}} \frac{\partial}{\partial y^{\beta}}\right) \Phi^{(0,0)}(y, 0, \xi, \eta)-\text { h.c. } \\
\mathcal{F}^{(1)(\ell, j)=}-\left(E^{\dot{\alpha}} \wedge E_{\dot{\alpha}}{ }^{\alpha} \frac{\partial}{\partial y^{\alpha}} \frac{\partial}{\partial \xi}+i E^{\alpha \dot{\alpha}} \wedge E_{\dot{\alpha}}{ }^{\beta} \frac{\partial}{\partial y^{\alpha}} \frac{\partial}{\partial y^{\beta}}\right) \Phi^{(\ell, j)}(y, 0, \xi, \eta)-\text { h.c. } \\
\nabla_{A} \Phi^{(\ell, j)}+i \Phi^{(\ell, j)} \star \bar{\pi}\left(Q_{A}\right)-i Q_{A} \star \Phi^{(\ell, j)}=0
\end{gathered}
$$

where the level decomposition of the higher spin field strength is given by

$$
\begin{gathered}
\mathcal{F}=\mathcal{F}^{(0,1 / 2)}+\sum_{\ell=1}^{\infty}\left(\mathcal{F}^{(\ell, 0)}+\mathcal{F}^{(\ell, 1 / 2)}\right), \\
\mathcal{F}^{(\ell, j)}=\nabla W^{(\ell, j)}+E \star W^{(\ell, j)}+W^{(\ell, j)} \star E
\end{gathered}
$$

and $\mathcal{F}^{(\ell, j)}$ has the same expansion in $(y, \bar{y}, \xi, \eta)$ as in $(3.5)$. 


\subsection{The $\mathcal{R}$-COnstraint}

In this section we show that the $O S p(1 \mid 4)$ valued curvature $\mathcal{R}$ subject to the constraint (3.8) describes the supergravity multiplet residing at level $(0,0)$.

From (3.8) and eqs. (2.35)-(2.37) it follows that up to first order in the weak field expansion the superspace torsion and Riemann tensor are given by

$$
\begin{aligned}
T^{\alpha \dot{\alpha}} & =\frac{i}{2} E^{\alpha} \wedge E^{\dot{\alpha}} \\
T^{\alpha} & =-2 E^{\dot{\alpha}} \wedge E_{\dot{\alpha}}{ }^{\alpha}-2 E^{\beta \dot{\alpha}} \wedge E_{\dot{\alpha}}^{\gamma} \Psi^{\alpha}{ }_{\beta \gamma}, \\
R^{\alpha \beta} & =i E^{\alpha} \wedge E^{\beta}+2 i E^{\dot{\alpha}} \wedge E_{\dot{\alpha}}{ }^{\gamma} \Psi^{\alpha \beta}{ }_{\gamma}-4 E^{\alpha \dot{\alpha}} \wedge E_{\dot{\alpha}}{ }^{\beta}-2 E^{\gamma \dot{\alpha}} \wedge E_{\dot{\alpha}}{ }^{\delta} C^{\alpha \beta}{ }_{\gamma \delta},
\end{aligned}
$$

where the gravitino curvature and Weyl tensor are defined by

$$
\begin{aligned}
\Psi_{\alpha \beta \gamma} & \left.\equiv \frac{\partial^{3}}{\partial y^{\alpha} \partial y^{\beta} \partial y^{\gamma}} \frac{\partial}{\partial \xi} \Phi\right|_{Y=\xi=\eta=0}, \\
C_{\alpha \beta \gamma \delta} & \left.\equiv \frac{\partial^{4}}{\partial y^{\alpha} \partial y^{\beta} \partial y^{\gamma} \partial y^{\delta}} \Phi\right|_{Y=\xi=\eta=0} .
\end{aligned}
$$

The Weyl tensor is the spinor derivative of the gravitino curvature, as given in (3.28). The constraints (3.13)-(3.15) describe the on-shell $\mathcal{N}=1$ pure supergravity multiplet with cosmological constant. To see this, we note that (3.13) and (3.14) contain the constraints:

$$
T_{\underline{\alpha \beta}}^{c}=-i\left(\Gamma^{c}\right)_{\underline{\alpha \beta}}, \quad T_{A b}^{c}=0,
$$

where $\underline{\alpha}=(\alpha, \dot{\alpha})$, which describe the $\mathcal{N}=1$ off-shell supergravity multiplet in superspace with auxiliary fields given by a complex scalar field $S+i P$ and a real vector [16] (see also [17]). The remaining constraints in (3.13)-(3.15) amount to setting the auxiliary vector equal to zero and the pseudo-scalar $P$ equal to a constant (in units where the $\mathrm{AdS}_{4}$ radius is set equal to 1).

\subsection{The $\mathcal{F}^{(1)}$-CONSTRAINT}

In this section we analyze the constraint (3.9) and show that their $\theta$-expansion contains the higher spin multiplets. 
The $A B=\alpha \beta$ and $A B=a \beta$ components of (3.9) read

$$
\mathcal{F}_{\underline{\alpha \beta}}^{(1)} \equiv 2 \nabla_{(\underline{\alpha}} W_{\underline{\beta})}-i\left(\Gamma^{c}\right)_{\underline{\alpha \beta}} W_{c}-2 i\left\{Q_{(\underline{\alpha}}, W_{\underline{\beta})}\right\}_{\star}=0
$$

and

$$
\begin{aligned}
\mathcal{F}_{a \beta}^{(1)} & \equiv \nabla_{a} W_{\beta}-\nabla_{\beta} W_{a}-\frac{1}{2}\left(\sigma_{a}\right)_{\beta} \dot{\gamma} W_{\dot{\gamma}}-i\left[P_{a}, W_{\beta}\right]_{\star}+i\left[Q_{\beta}, W_{a}\right]_{\star} \\
& =-\frac{1}{4}\left(\sigma_{a}\right)_{\beta} \dot{\gamma} \frac{\partial}{\partial \bar{y} \dot{\gamma}} \frac{\partial}{\partial \xi} \Phi(0, \bar{y}, \xi, \eta) \star \Gamma,
\end{aligned}
$$

where the level index $(\ell, j)$ has been suppressed. The structure of these constraints is similar to that of ordinary Yang-Mills theory in $\mathcal{N}=1$ superspace, and we may proceed in an analogous fashion in order to show that no new component fields arise upon $\theta$-expanding $W_{\alpha}$ and $W_{a}$.

First, from $\delta W_{\alpha}=\nabla_{\alpha} \epsilon$, where $\epsilon$ is the $h s(1 \mid 4)$ valued gauge parameter, it follows that we can fix a gauge in which

$$
W_{\alpha} \mid=0
$$

From (3.19) and (3.21) it follows that

$$
\nabla_{(\underline{\alpha}} W_{\underline{\beta})}\left|=\frac{i}{2}\left(\Gamma^{c}\right)_{\underline{\alpha \beta}} W_{c}\right|
$$

By using the $\theta^{2}$-component of $\epsilon$, i.e. $\left(\nabla_{\underline{\alpha}} \nabla_{\underline{\beta}}-\nabla_{\underline{\beta}} \nabla_{\underline{\alpha}}\right) \epsilon \mid$, we can impose the further gauge condition:

$$
\left(\nabla_{\underline{\alpha}} W_{\underline{\beta}}-\nabla_{\underline{\beta}} W_{\underline{\alpha}}\right) \mid=0
$$

Hence the first non-trivial components in the $\theta$-expansion of $W_{\alpha}$ are the leading components of $W_{a}$, i.e. $W_{a} \mid$. We proceed by examining $\nabla_{\alpha} W_{b} \mid$. From (3.20) and (3.21), (3.22) and (3.23) it follows that

$\nabla_{\alpha} W_{b}\left|=i\left[Q_{\alpha}, W_{b} \mid\right]_{\star}-i \psi_{b}^{\dot{\beta}}\left(\sigma^{a}\right)_{\dot{\beta} \alpha} W_{a}\right|+\frac{1}{4}\left(\sigma_{b}\right)_{\alpha}{ }^{\dot{\beta}} \frac{\partial}{\partial \bar{y}^{\dot{\beta}}} \frac{\partial}{\partial \xi} \Phi^{(\ell, j)}(0, \bar{y}, \xi, \eta) \mid \star \Gamma$ 
where $\psi_{a}{ }^{\alpha}=E_{a}{ }^{\alpha} \mid$. In order to evaluate the last term in (3.24), we examine the $A B=$ $a b$ component of (3.9). This constraint has the same form as in the formulation in ordinary spacetime. The latter have been analyzed in detail in [18]. Using these results we conclude that the last term in (3.24) can be written in terms of spacetime derivatives of $W_{a} \mid$. More specifically, the gauge fields $W_{a, r}(p, q)$ with $|p-q| \leq 1$ are independent, while those with $|p-q| \geq 2$ are auxiliary fields that are $\left[\frac{1}{2}|p-q|\right]$ spacetime derivatives of the independent gauge fields. Moreover, it follows that the spin $s$ field strengths contained in $\Phi^{(\ell, j)}(0, \bar{y}, \xi, \eta)$ can be written as $[s]$ spacetime derivatives of the independent gauge fields. Combining the above results we deduce that $W_{A}$ can be $\theta$-expanded in terms of $W_{a} \mid$ and its spacetime derivatives.

It is also possible to eliminate the auxiliary fields in a manifestly superspace covariant manner. To this end, we first use (3.19) to solve for $W_{a}$ in terms of $W_{\alpha}$ :

$$
\left.W_{a}=\frac{i}{2}\left(\Gamma_{a}\right) \frac{\alpha \beta}{\left(\nabla_{\underline{\alpha}}\right.} W_{\underline{\beta}}-i\left\{Q_{\underline{\alpha}}, W_{\underline{\beta}}\right\}_{\star}\right)
$$

Inserting this into (3.20) we then obtain

$$
\begin{aligned}
& i\left[P_{a}, W_{\beta}\right]_{\star}-\frac{i}{2}\left(\Gamma_{a}\right) \frac{\gamma \delta}{\gamma^{\prime}}\left\{Q_{\beta},\left\{Q_{\underline{\gamma}}, W_{\underline{\delta}}\right\}_{\star}\right\}_{\star} \\
= & \nabla_{a} W_{\beta}-\frac{i}{2}\left(\Gamma_{a}\right) \frac{\gamma \delta}{-} \nabla_{\beta}\left(\nabla_{\underline{\gamma}} W_{\underline{\delta}}-i\left\{Q_{\underline{\gamma}}, W_{\underline{\delta}}\right\}_{\star}\right)-\frac{1}{2}\left(\Gamma_{a}\right) \frac{\gamma \delta}{\underline{\gamma}}\left\{Q_{\beta}, \nabla_{\underline{\gamma}} W_{\underline{\delta}}\right\}_{\star} \\
& -\frac{1}{2}\left(\sigma_{a}\right)_{\beta} \dot{\gamma} W_{\dot{\gamma}}+\frac{1}{4}\left(\sigma_{a}\right)_{\beta} \dot{\gamma} \frac{\partial}{\partial \bar{y} \dot{\gamma}} \frac{\partial}{\partial \xi} \Phi^{(\ell, j)}(0, \bar{y}, \xi, \eta) \star \Gamma .
\end{aligned}
$$

Expanding in $y$ and $\bar{y}$, the $(m, n)$ component of the above equations are supposed to be solved for $W_{\beta}(m+1, n-1)$ in terms of $W_{\beta}\left(m^{\prime}, n^{\prime}\right)$ with $\left|m^{\prime}-n^{\prime}\right|<|m-n|$ covered by various combinations of spinor and vector derivatives (the structure is similar to that of the $A B=a b$ components of (3.9)). Hence a subset of the $W_{\alpha}(m, n)$ are independent gauge superfields, and the remaining $W_{\alpha}(m, n)$ and all of $W_{a}$ are auxiliary superfields. The details of this, and in particular, the constraints obeyed by the independent gauge superfields, remain to be worked out.

\subsection{The $\Phi$-CONSTRAINT}

In this section we show that the master field $\Phi$ subject to the constraint (3.10) contains the physical scalar multiplet at level $(-1,1 / 2)$ and the field strengths (i.e. the Weyl tensors) of the higher level physical gauge fields. The $A=a$ component 
of (3.10) has the same structure as in the formulation in ordinary spacetime. Hence the components $\Phi_{r}^{(\ell, j)}(k, k+q)$ with $k>0$ are auxiliary fields which can be expressed as $k$ bosonic derivatives of the components $\Phi_{r}^{(\ell, j)}(0, q)$.

\section{The field strength multiplets}

Eq. (3.9) identifies $\Phi_{r}^{(\ell, j)}(0, q)$ for $\ell \geq 0, j=0,1 / 2, q=4 \ell+3+2 j+r$ with the non-vanishing spin $s=q / 2$ field strengths discussed in Section 3.2 and 3.3.

It is remarkable that (3.10) also yields the on-shell constraints on the superfield $\Phi_{0 \dot{\alpha}_{1} \ldots \dot{\alpha}_{2 s}}^{(\ell, j)}(s=2 \ell+3 / 2+j)$ whose $\theta$-expansion yields the level $(\ell, j)$ field strength supermultiplet. We find these constraints to be:

$$
\begin{aligned}
\nabla_{\beta} \Phi_{0 \dot{\alpha}_{1} \ldots \dot{\alpha}_{2 s}}^{(\ell, j)} & =0 \\
\nabla_{\dot{\beta}} \Phi_{0 \dot{\alpha}_{1} \ldots \dot{\alpha}_{2 s}}^{(\ell, j)} & =(-1)^{2 s} \Phi_{1 \dot{\beta} \dot{\alpha}_{1} \ldots \dot{\alpha}_{2 s}}^{(\ell, j)}, \\
\nabla_{\dot{\beta}} \Phi_{1 \dot{\alpha}_{1} \ldots \dot{\alpha}_{2 s+1}}^{(\ell, j)} & =-i(-1)^{2 s}(2 s+1) \epsilon_{\dot{\beta}_{\left(\dot{\alpha}_{1}\right.}} \Phi_{\left.0 \dot{\alpha}_{2} \ldots \dot{\alpha}_{2 s+1}\right)}^{(\ell, j)},
\end{aligned}
$$

where

$$
\Phi_{r \dot{\alpha}_{1} \ldots \dot{\alpha}_{q}}^{(\ell, j)}=\left.\frac{\partial^{q}}{\partial \bar{y}^{\dot{\alpha}_{1}} \cdots \partial \bar{y}^{\dot{\alpha}_{q}}} \Phi_{r}^{(\ell, j)}(0, q)\right|_{Y=0}
$$

The independent superfield is evidently $\Phi_{0 \dot{\alpha}_{1} \ldots \dot{\alpha}_{2 s}}^{(\ell, j)}$. Indeed we can eliminate $\Phi_{1 \dot{\alpha}_{1} \ldots \dot{\alpha}_{2 s+1}}^{(\ell, j)}$ from the symmetric part of (3.28). From (3.28) and (3.29) we then find

$$
\begin{aligned}
\nabla^{\dot{\beta}} \Phi_{0 \dot{\beta} \dot{\alpha}_{2} \ldots \dot{\alpha}_{2 s}}^{(\ell, j)} & =0 \\
\nabla_{\dot{\beta}} \nabla_{\left(\dot{\alpha}_{1}\right.} \Phi_{\left.0 \dot{\alpha}_{2} \ldots \dot{\alpha}_{2 s+1}\right)}^{(\ell, j)} & =-i(2 s+1) \epsilon_{\dot{\beta}_{\left(\dot{\alpha}_{1}\right.}} \Phi_{\left.0 \dot{\alpha}_{2} \ldots \dot{\alpha}_{2 s+1}\right)}^{(\ell, j)}
\end{aligned}
$$

Hence the complete set of constraints on $\Phi_{0 \dot{\alpha}_{1} \ldots \dot{\alpha}_{2 s}}^{(\ell, j)}$ in the first order in the weak field expansion are (3.27), (3.31) and (3.32).

The level $(-1,1 / 2)$ scalar multiplet

It remains to analyze $\Phi_{0}^{(-1,1 / 2)}(0,0)$ and $\Phi_{1}^{(-1,1 / 2)}(0,1)$ which contain the superfields 


$$
\left.\boldsymbol{\phi} \equiv \Phi\right|_{Y=\xi=\eta=0}, \quad \Psi_{\dot{\alpha}} \equiv-\left.\frac{\partial}{\partial \xi} \frac{\partial}{\partial \bar{y}^{\dot{\alpha}}} \Phi\right|_{Y=\xi=\eta=0}
$$

From the $A=a$ components of (3.10) it follows that the leading components $\phi=\phi \mid$ and $\psi_{\dot{\alpha}}=\Psi_{\dot{\alpha}} \mid$ obey physical field equations with masses $m_{\phi}^{2}=-2$ and $m_{\psi}=0$. The $A=\alpha$ and $A=\dot{\alpha}$ components of (3.10) yields

$$
\nabla_{\alpha} \phi=0, \quad \nabla_{\dot{\alpha}} \phi=\Psi_{\dot{\alpha}}, \quad \nabla_{\dot{\beta}} \nabla_{\dot{\alpha}} \phi=-i \epsilon_{\dot{\beta} \dot{\alpha}} \phi
$$

which are the appropriate constraints for the on-shell scalar multiplet. Note that, in the presence of cosmological constant, the quantity $\nabla_{\dot{\alpha}} \nabla_{\dot{\beta}} \phi$, where $\phi$ is chiral, can contain both $\phi$ and $\phi^{\dagger}$ terms.

In summary, the only physical superfield that arises in $\Phi$ is $\boldsymbol{\phi}$, which describes the scalar multiplet residing at level $(-1,1 / 2)$ in Table 1.

\section{Comments}

In this paper we have given the $\mathcal{N}=1$ superspace formulation of the massless $4 \mathrm{D}$ higher spin gauge theory based on the minimal $\mathcal{N}=1$ higher spin algebra $h s(1 \mid 4)$. The general arguments for the equivalence between the superspace formulation and the formulation in ordinary spacetime are given at the end of Section 2.1, and explicitly verified in Section 3.

In the first order in the weak field expansion (see Section 3.1), the resulting on-shell constraints on the supergravity multiplet are given in (3.13), (3.14) and (3.15), those on the higher spin field strengths in (3.27), (3.31) and (3.32), and those on the scalar multiplet in (3.34). The supergravity constraints describe the pure supergravity field equations without higher derivative corrections and with cosmological constant. The corrections to these equations, including the stress-energy from the scalar and higher spin multiplets as well as higher order curvature corrections, can be obtained order by order in the covariant weak field expansion scheme, as discussed for example in [11].

The master constraints (2.13) and (2.14) generalize straightforwardly to the supersymmetric higher spin gauge theories based on the $\mathcal{N} \geq 2$ extended higher spin algebras discussed in [4]. In all these algebras the scalar master field $\widehat{\Phi}$ obeys a reality condition of the form $\widehat{\Phi}^{\dagger}=\pi(\widehat{\Phi}) \star \Gamma$, where $\Gamma$ is a suitable operator formed out of the fermionic oscillators used in the construction of the extended higher spin 
algebras. The equivalence between the resulting superspace formulations and the previous formulations in bosonic spacetime follows from the general argument given at the end of Section 2.1.

It is well-known that the $\mathcal{N}=8$ supergravities in $D=4$ exhibit hidden symmetries. In the superspace formulation, these symmetries arise naturally as a result of embedding the 28 vector fields into the composite $S U(8)$ connection in superspace $[19,20]$. The required torsion constraints also arise in the leading order in the weak field expansion of the $\mathcal{N}=8$ higher spin gauge theory, as a subset of the constraints on the $O S p(8 \mid 4)$ valued curvature $\mathcal{R}$ (see Section 3.2 for the analogous discussion in the case of $\mathcal{N}=1$ ). There are, of course, corrections to the torsion constraints, and it would be interesting to examine to what extent they affect the potential for the 70 supergravity scalars, $\phi_{i j k l}$. In principle, this potential can be computed directly by evaluating the scalar field equation order by order by in the curvature expansion scheme [11]. However, this procedure does not rely on the superspace formulation. To make use of the superspace formulation, one should first find the manifestly $S L(2, \mathbb{C}) \times S U(8)$ covariant formulation, i.e. generalize the identification of the exact spin connection $\Omega_{M}$ in (2.25) to include also the $S U(8)$-connection, and then examine the resulting corrected supergravity torsion constraints, in which terms depending on the scalars $\phi_{i j k l}$ (but not their derivatives) can give rise to corrections to the potential. Modulo such corrections, the potential for $\phi_{i j k l}$ in the higher spin gauge theory is given by the scalar potential of the supergravity theory.

It is straightforward to generalize the formalism described in this paper to obtain an $\mathcal{N}=4, D=5$ superspace formulation of the 5D HS gauge theory based on the $h s(2,2 \mid 4)$ algebra [21]. The massless spectrum consists of the supergravity multiplet and a tower of $s_{\max }=4,6,8, \ldots$ multiplets with spin range 4 . The superspace master fields are a zero-form $\Phi$ and a one-form $A_{M}$ which are expansions in terms of bosonic oscillators $y_{\alpha}$ and $\bar{y}_{\alpha}(\alpha=1, \ldots, 4)$ and fermionic $S U(4)$ oscillators $\xi^{i}$ and $\bar{\xi}_{i}(i=1, \ldots, 4)$ governed by the same $\tau$ and hermicity conditions as in [21]. The supertranslations, $Q_{A}=\left(P_{a}, Q_{\alpha}^{i}, Q_{\alpha i}\right)$ are given by $P_{a}=\left(\Gamma_{a}\right)^{\alpha \beta} y_{\alpha} \bar{y}_{\beta}, Q_{\alpha}^{i}=\xi^{i} \bar{y}_{\alpha}$ and $Q_{\alpha i}=\bar{\xi}_{i} y_{\alpha}$. Applying the superspace formalism described in this paper yields the following linearized $\Phi$-constraint:

$$
\nabla_{A} \Phi+Q_{A} \star \Phi-\Phi \star \pi\left(Q_{A}\right)=0
$$

where $\nabla_{A}$ is the covariant derivative in the rigid $\mathrm{AdS}_{5}$ superspace obeying $d \Omega+\Omega \star$ $\Omega=0$ and $\pi$ is defined in [21]. The above constraint is integrable and consistent with the $(y, \bar{y}, \xi, \bar{\xi})$-expansion of $\Phi$. As shown in [21], the $A=a$ component of (4.1) yields the correct linearized field equations for the $h s(2,2 \mid 4)$ theory. We expect that the $A=\alpha i$ components of (4.1) contain the corresponding superspace constraints. 
Assuming that the $h s(2,2 \mid 4)$ theory is related to the $1 / N^{2}$ expansion of the free $S U(N)$ SYM theory in $\mathcal{N}=4, d=4$ superspace, the supergravity multiplet couples to the superconformal current and the $s_{\max }=4,6, \ldots$ multiplets couple to the higher spin supercurrents of the free SYM theory. Hence, the reduction of the $\Phi$-constraint to $d=4, \mathcal{N}=4$ superspace must reproduce the constrained superfield strengths of the prepotentials coupling to the currents of the free SYM theory.

The superspace formulation of ordinary supergravity is indispensable in their coupling to extended objects, which are described by $\kappa$-symmetric brane actions. It would be interesting to see if the superspace formulation of the HS gauge theories presented in this paper could be utilized for their coupling to extended objects, which in turn might provide a dual description of the spacetime physics at large energies.

Finally, the general argument given at the end of Section 2.1 suggests further extensions of the 4D (super)spacetime by extra coordinates, possibly infinitely many, corresponding to a suitable coset of the HS algebra and with the property that a finite number of independent dynamical fields contain all component fields in the master fields in their expansion in the extra coordinates. Free field constructions of this type have been considered in [8, 22].

\section{ACKNOWLEDGEMENTS}

E.S. would like to thank ITP in Uppsala University, and P.S. would like to thank the George P. and Cynthia W. Mitchell Institute for Fundamental Physics, for hospitality. The work of E.S. is supported in part by NSF Grant PHY-0070964. 


\section{A Notations and Conventions}

We use the following notation for expanding functions of the oscillators $y$ and $\bar{y}$ :

$$
\begin{aligned}
f(y, \bar{y}) & =\sum_{m, n=0}^{\infty} f(m, n) \\
f(m, n) & =\frac{1}{m ! n !} f_{\alpha_{1} \ldots \alpha_{m}, \dot{\alpha}_{1} \ldots \dot{\alpha}_{n}} y^{\alpha_{1}} \cdots y^{\alpha_{m}} \bar{y}^{\dot{\alpha}_{1}} \cdots \bar{y}^{\dot{\alpha}_{n}}
\end{aligned}
$$

Differentiation with respect to the fermionic oscillators is defined by

$$
\frac{\partial \widehat{f}}{\partial \xi}=\frac{1}{2}[\xi, \widehat{f}]_{\star} .
$$

The van der Waerden symbols are defined by

$$
\begin{aligned}
\left(\sigma^{a} \bar{\sigma}^{b}\right)_{\alpha \beta} & =\eta^{a b} \epsilon_{\alpha \beta}+\left(\sigma^{a b}\right)_{\alpha \beta} \\
\frac{1}{2} \epsilon^{a b c d} \sigma_{c d} & =i \sigma^{a b} \\
\left(\bar{\sigma}^{a b}\right)_{\dot{\alpha} \dot{\beta}} & =\left(\left(\sigma^{a b}\right)_{\alpha \beta}\right)^{\dagger}
\end{aligned}
$$

where $\left(\bar{\sigma}^{a}\right)_{\dot{\alpha} \beta}=\left(\left(\sigma^{a}\right)_{\alpha \dot{\beta}}\right)^{\dagger}=\left(\sigma^{a}\right)_{\beta \dot{\alpha}}, \epsilon_{\alpha \beta} \epsilon^{\gamma \delta}=2 \delta_{[\alpha}^{\gamma} \delta_{\beta]}^{\delta}, \epsilon_{\dot{\alpha} \dot{\beta}}=\left(\epsilon_{\alpha \beta}\right)^{\dagger}$, and spinor contractions are according to north-west-south-east rule. The completeness relations are given by

$$
\begin{array}{ll}
\left(\sigma^{a}\right)_{\alpha \dot{\alpha}}\left(\sigma_{a}\right)_{\beta \dot{\beta}}=-2 \epsilon_{\alpha \beta} \epsilon_{\dot{\alpha} \dot{\beta}}, & \left(\sigma^{a b}\right)_{\alpha \beta}\left(\sigma_{a b}\right)_{\gamma \delta}=-8 \epsilon_{\alpha(\gamma} \epsilon_{\delta) \beta} \\
\left(\sigma^{a}\right)^{\alpha \dot{\alpha}}\left(\sigma^{b}\right)_{\alpha \dot{\alpha}}=-2 \eta^{a b}, & \left(\sigma^{a b}\right)^{\alpha \beta}\left(\sigma_{c d}\right)_{\alpha \beta}=4 \delta_{c d}^{a b}-2 i \epsilon_{c d}^{a b} .
\end{array}
$$

The $O S p(1 \mid 4)$ generators in spinor basis are given by

$$
Q_{\alpha}=\frac{1}{2} y_{\alpha} \xi, \quad M_{\alpha \beta}=y_{\alpha} y_{\beta}, \quad P_{\alpha \dot{\alpha}}=y_{\alpha} \bar{y}_{\dot{\alpha}}
$$

and they obey 


$$
\begin{array}{llll}
\left\{Q_{\alpha}, Q_{\beta}\right\} & =\frac{1}{2} M_{\alpha \beta}, & \left\{Q_{\alpha}, Q_{\dot{\alpha}}\right\} & =\frac{1}{2} P_{\alpha \dot{\alpha}}, \\
{\left[Q_{\alpha}, M_{\beta \gamma}\right]} & =4 i \epsilon_{\alpha(\beta} Q_{\gamma)}, & {\left[Q_{\alpha}, P_{\beta \dot{\beta}}\right]=2 i \epsilon_{\alpha \beta} Q_{\dot{\beta}},} \\
{\left[M_{\alpha \beta}, M_{\gamma \delta}\right]=4 i\left(\epsilon_{\alpha(\gamma} M_{\delta) \beta}+\epsilon_{\beta(\gamma} M_{\delta) \alpha}\right),} & {\left[P_{\alpha \dot{\alpha}}, M_{\beta \gamma}\right]=4 i \epsilon_{\alpha(\beta} P_{\gamma) \dot{\alpha}},} \\
{\left[P_{\alpha \dot{\alpha}}, P_{\beta \dot{\beta}}\right]=2 i\left(\epsilon_{\alpha \beta} M_{\dot{\alpha} \dot{\beta}}+\epsilon_{\dot{\alpha} \dot{\beta}} M_{\alpha \beta}\right) .} &
\end{array}
$$

The $S O(3,2)$ commutation relations are $\left[M_{\hat{a} \hat{b}}, M_{\hat{c} \hat{d}}\right]=-i\left(\eta_{\hat{b} \hat{c}} M_{\hat{a} \hat{d}}+3\right.$ more $)$, where $\eta_{\hat{a} \hat{b}}=\operatorname{diag}(-+++-)$ and

$$
\begin{aligned}
M_{a b} & =\frac{1}{8}\left(\sigma_{a b}\right)^{\alpha \beta} M_{\alpha \beta}+\text { h.c. }, & P_{a} & =M_{a 5}=\frac{1}{4}\left(\sigma_{a}\right)^{\alpha \dot{\alpha}} P_{\alpha \dot{\alpha}}, \\
M_{\alpha \beta} & =\left(\sigma^{a b}\right)_{\alpha \beta} M_{a b}, & P_{\alpha \dot{\alpha}} & =-2\left(\sigma^{a}\right)_{\alpha \dot{\alpha}} P_{a} .
\end{aligned}
$$

An $S O(3,2)$ valued element $\Lambda$ is expanded as

$$
\Lambda=\frac{1}{2} \Lambda^{a b} M_{a b}+\Lambda^{a} P_{a}=\frac{1}{4} \Lambda^{\alpha \beta} M_{\alpha \beta}+\text { h.c. }+\Lambda^{\alpha \dot{\alpha}} P_{\alpha \dot{\alpha}}
$$

where

$$
\begin{aligned}
\Lambda_{a b} & =\frac{1}{2}\left(\sigma_{a b}\right)^{\alpha \beta} \Lambda_{\alpha \beta}+\text { h.c. }, & \Lambda_{a} & =-2\left(\sigma_{a}\right)^{\alpha \dot{\alpha}} \Lambda_{\alpha \dot{\alpha}} \\
\Lambda_{\alpha \beta} & =\frac{1}{4}\left(\sigma^{a b}\right)_{\alpha \beta} \Lambda_{a b}, & \Lambda_{\alpha \dot{\alpha}} & =\frac{1}{4}\left(\sigma^{a}\right)_{\alpha \dot{\alpha}} \Lambda_{a} .
\end{aligned}
$$

We use the following superspace conventions:

$$
\begin{aligned}
G & =\frac{1}{p !} E^{A_{1}} \cdots E^{A_{p}} G_{A_{p} \ldots A_{1}}=\frac{1}{p !} d X^{M_{1}} \cdots d X^{M_{p}} G_{M_{p} \ldots M_{1}} \\
d G & =\frac{1}{p !} d X^{M_{1}} \cdots d X^{M_{p}} d X^{N} \frac{\partial}{\partial X^{N}} G_{M_{p} \ldots M_{1}} .
\end{aligned}
$$

The right-action of the exterior derivative implies that $d(G H)=G d H+(-1)^{q}(d G) H$ for a $p$-form $G$ and $q$-form $H$. The hermitian conjugation of $G H$ is

$$
(G H)^{\dagger}=(-1)^{p q} H^{\dagger} G^{\dagger}=(-1)^{\epsilon(G) \epsilon(H)} G^{\dagger} H^{\dagger}
$$

The conversion between Lorentz and spinor indices for the vector components of a one-form $G=E^{A} G_{A}$ is given by 


$$
\begin{aligned}
E^{a} & =-2\left(\sigma^{a}\right)_{\alpha \dot{\alpha}} E^{\alpha \dot{\alpha}}, \quad G_{a}=\frac{1}{4}\left(\sigma_{a}\right)^{\alpha \dot{\alpha}} G_{\alpha \dot{\alpha}} \\
E^{\alpha \dot{\alpha}} & =\frac{1}{4}\left(\sigma^{a}\right)_{\alpha \dot{\alpha}} E_{a}, \quad G_{\alpha \dot{\alpha}}=-2\left(\sigma^{a}\right)_{\alpha \dot{\alpha}} G_{a} .
\end{aligned}
$$

The Lorentz covariant derivatives are defined by:

$$
\begin{aligned}
& \nabla V^{\alpha}=d V^{\alpha}+V^{\beta} \Omega_{\beta}{ }^{\alpha} \\
& \nabla V^{a}=d V^{a}+V^{b} \Omega_{b}{ }^{a}
\end{aligned}
$$

where $\Omega_{a b}$ and $\Omega_{\alpha \beta}$ are related to each other as in (A.11). The graded commutator of two covariant derivatives is given by

$$
2 \nabla_{[A} \nabla_{B]} V^{C}=(-1)^{D(A+B)} V^{D} R_{A B, D}^{C}-T_{A B}{ }^{D} \nabla_{D} V^{C}
$$

and the torsion identity reads

$$
\nabla_{[A} T_{B C]}^{D}+T_{[A B \mid}^{E} T_{E \mid C]}^{D}=R_{[A B, C]}^{D} .
$$

The non-vanishing rigid $\mathrm{AdS}_{4}$ superspace torsions and curvatures are

$$
\begin{aligned}
T_{\alpha \dot{\beta}}{ }^{c} & =-i\left(\sigma^{c}\right)_{\alpha \dot{\beta}}, \quad T_{a \dot{\beta}}{ }^{\gamma}=-\frac{1}{2}\left(\sigma_{a}\right)_{\dot{\beta}}{ }^{\gamma}, \\
R_{\alpha \beta}{ }^{\gamma \delta} & =2 i \delta_{(\alpha}^{\gamma} \delta_{\beta)}^{\delta}, \quad R_{a b}{ }^{\gamma \delta}=\frac{1}{2}\left(\sigma_{a b}\right)^{\gamma \delta} .
\end{aligned}
$$

Our conventions are such that a scalar field $\phi$ with mass $m$ and AdS energy $E$ obeys

$$
\left(\nabla^{2}-m^{2}\right) \phi=0, \quad E(E-3)=m^{2} R^{2}
$$

where $R$ is the AdS radius, which we have set equal to 1. 


\section{References}

[1] M.A. Vasiliev, Properties of equations of motion of interacting gauge fields of all spins in (3+1) dimensions, Class. Quant. Grav. 8 (1991) 1387

[2] E. Sezgin and P. Sundell, Higher spin N=8 supergravity, JHEP 9811 (1998) 016, hep-th/9805125.

[3] E. Sezgin and P. Sundell, Massless higher spins and holography, Nucl. Phys. B644 (2000) 303, hep-th/0205131

[4] J. Engquist, E. Sezgin and P. Sundell, On $\mathcal{N}=1,2,4$ higher spin gauge theories in four dimensions, hep-th/0207101

[5] I.R. Klebanov and A. M. Polyakov, AdS dual of the critical $O(N)$ vector model, hep-th/0210114

[6] B. Sundborg, Stringy gravity, interacting tensionless strings and massless higher spins, Nucl. Phys. Proc. Suppl. 102 (2001) 113, hep-th/0103247.

[7] E. Sezgin and P. Sundell, Doubletons and 5D higher spin gauge theory, JHEP 0109 (2001) 036, hep-th/0105001.

[8] M.A. Vasiliev, Conformal higher spin symmetries of $4 d$ massless supermultiplets and osp(L,2M) invariant equations in generalized (super)space, Phys. Rev. D66 (2002) 066006, hep-th/0106149

[9] E. Witten, talk given at J.H. Schwarz' 60th Birthday Conference, Cal Tech, Nov 2-3, 2001.

[10] M.A. Vasiliev, Dynamics of massless higher spins in the second order in curvatures, Phys. Lett. B238 (1990) 305

[11] E. Sezgin and P. Sundell, Analysis of higher spin field equations in four dimensions, JHEP 0207 (2002) 055, hep-th/0205132

[12] S.J. Gates Jr., S.M. Kuzenko and A.G. Sibiryakov, Towards a unified theory of massless superfields of all superspins, Phys. Lett. B394 (1997) 343, hep-th/9611193

[13] S.E. Konstein and M.A. Vasiliev, Massless representations and admissibility condition for higher spin superalgebras, Nucl. Phys. B312 (1989) 402

[14] M.A. Vasiliev, Algebraic aspects of the higher spin problem, Phys. Lett. B257 (1991) 111 
[15] M.A. Vasiliev, Higher spin gauge theories: Star-product and AdS space, (1999), hep-th/9910096

[16] R. Grimm, J. Wess and B. Zumino, A complete solution of the Bianchi identities in superspace, Nucl. Phys. B152 (1979) 255

[17] J. Bagger and J. Wess, Supersymmetry and supergravity, (Princeton University Press, 1983)

[18] M.A. Vasiliev, Free massless fields of arbitrary spin in the de sitter space and initial data for a higher spin superalgebra, Fortsch. Phys. 35 (1987) 741

[19] L. Brink and P.S. Howe, The $N=8$ supergravity in superspace, Phys. Lett. B88 (1979) 268

[20] P.S. Howe and H. Nicolai, Gauging N=8 supergravity in superspace, Phys. Lett. B109 (1982) 269

[21] E. Sezgin and P. Sundell, Towards massless higher spin extension of D $D=5$, $N=8$ gauged supergravity, JHEP 0109 (2001) 025, hep-th/0107186

[22] C. Devchand and J. Nuyts, Lorentz covariance, higher-spin superspaces and self-duality, AIP Conf. Proc. 453 (2002) 317 